Historic, archived document

Do not assume content reflects current scientific knowledge, policies, or practices. 



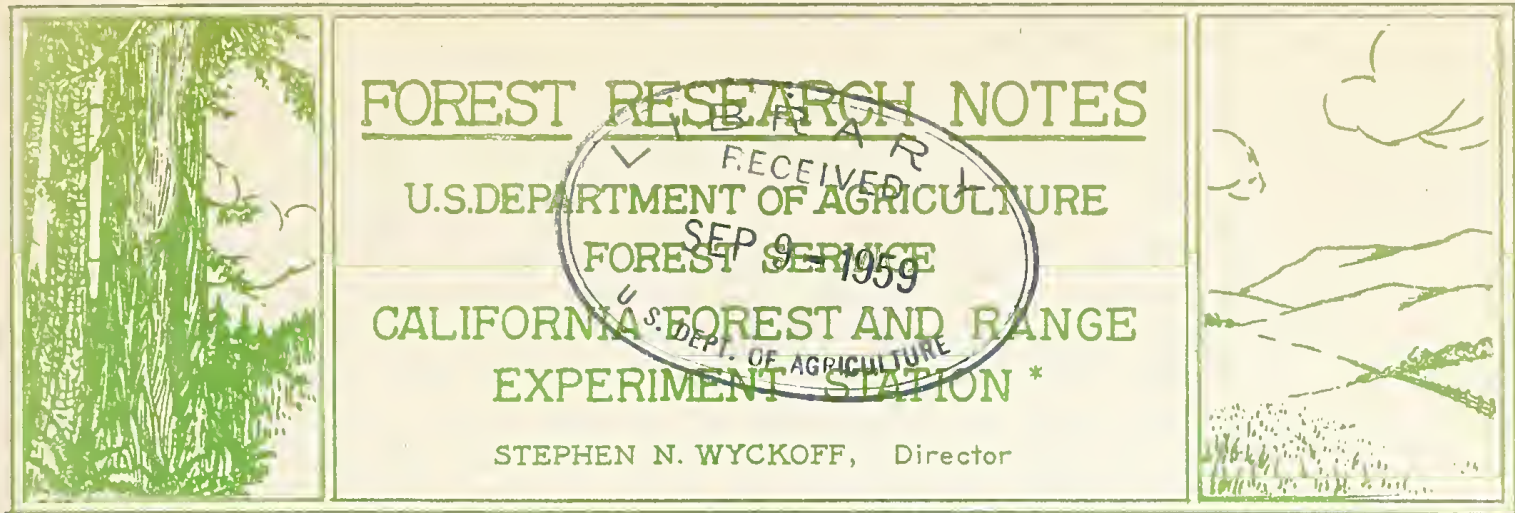

No. 66

February 6, 1950

\section{BOARD-FOOT AND CUBIC-FOOT VOLUME TABLES FOR SECOND-GROWTH REDWOOD}

By A. A. Hasell/

Forest Survey, Division of Forest Economics

The tables presented here are for use in estimating the volume of second-growth redwood trees: board-foot volume by the Scribner and International $1 / 4 "$ rules, cubic volume in the sawlog portion, and cubic volume to a 4 " top diameter. The tables were prepared in connection with the forest survey of California, which is being conducted as part of the nation-vide Forest Survey Project.

Since the tables by Bruce and Schumacher2/ were prepared, sufficient time has elapsed to provide larger trees for measurement. The volumes given here for sawlog-size trees are based on measurements on 118 trees, of which 70 represent new data mainly in the larger size classes. The largest tree measured was 60 inches $d . b . h$, and the tables extend through this diameter class. For second-growth trees larger than 60 inches $d . b . h$. it is likely that existing old-growth tables will apply satisfactorily. Data for trees of less than sawlog size were randomly selected from Forest Service data collected in 1899. All data were collected in Del Norte, Humbolat, Mendocino, and Sonoma Counties. The top utilization for the sawlog portion of the trees was determined from a survey of utilization practices on Forest Service sales (fig. 3) in other timber types, since an adequate sample of practice in secondgrowth redwood was not available.

I) Crateful acknowledgment is made of assistance given by other members of the Division of Forest Economics, including Miss A. E. Rae for computations, $K$. Schoenlank for compilation of volumes, and $E$. M. Hornibrook for helpful advice.

2/ 1925. Bruce, Donald, and Francis X. Schumacher. Revised Volume Tables for Second-growth Redwood. Jour. Forestry 23: 148-155. 


\section{Procedure}

Tree measurements were plotted on Forest Service Tree Measurement Form 558a (Rev. November 1928) and cubic volumes were obtained by planimetering. Board-foot volumes were obtained by scaling in 16-foot lengths, allowing 0.3-foot for trim. Additional top sections and small trees were scaled as 8-foot logs or fractions of 8-foot logs. Diameters at the small end were read to 0.I-inch on Form 558a, and the scale was determined from the following formulas:

16-foot logs

Scribner volume $=0.79 D^{2}-2 D-4$

International $1 / 4^{\prime \prime}$ volume $=0.796 \mathrm{D}^{2}-1.375 \mathrm{D}-1.230$

$\underline{8 \text {-foot logs }}$

Scribner volume $=0.395 D^{2}-D-2$
International $1 / 4 "$ volume $=\left(0.44 D^{2}-1.2 D-0.3\right) 0.904762$

The volume tables are based on the relationship of volume to diameter, height, and the Girard form class, which may be defined as d.i.b. at the small end of the butt 16-foot log expressed as a percentage of d.b.h. o.b.

\section{Converting Factors}

The volumes are tabulated for Form Class 70, which is average for the data, but factors are given for converting volumes to other form classes. The tables can therefore be readily adapted or localized to particular stands, or types of trees in the stand, by determining the form class of the particular stand to be cruised. Determination of form class on 50 trees, well distributed throughout the range of diameters and over the area, should be satisfactory for this purpose. If form class does not vary with diameter, the stand average may be used.

Heights to tip and to 4-, 8-, 10-, and 12-inch i.b. top diameters are curved over d.b.h. in Figure 1. These curves indicate average taper in the tops and therefore provide a guide to converting heights from one top diameter basis to another. Together with the curve of merchantable length in Figure 2, they make it possible to adjust the total cubic table to larger top diameters, or to approximate the board-foot volumes on a fixed top diameter basis.

\section{Growth and Form-class Determinations}

The bark thickness curves in Figures 4, 5, and 6 are useful in connection with growth studies and form-class determination.

Example of growth determination: 
(1) Tree 30.0 in. d.b.h., 5 logs, present volume 1000 b.f. Scribner. Increment core shows $1.5^{\prime \prime}$ radial growth past 10 years.

(2) Fig. 4 indicates bark thickness now 4.8". Then, 30.0 $4.8-2(1.5)=22.2$ in. d.i.b. ten years ago.

(3) Fig. 6 indicates bark thickness of 4.2 in. 10 years ago, so $22.2+4.2=26.4$ in. $d . b . h$. 10 years ago.

(4) Assume curve of height-diameter relationship indicates height of 4 logs to merchantable top for tree 26.4 in. d.b.h. From Table 1, volume 10 years ago is $615+(710-615) 1 / 5=634 \mathrm{~b} . f$.

(5) Growth during past 10 years was $1000-634=366$ board feet.

Example of form class determination:

(1) D.B.H. of tree is $30.0 \mathrm{in.}$ o.b.

(2) Determine location of top of first 16-foot log (1.5' stump on uphill side plus $16.0^{\prime}$ log plus $0.3^{\prime}$ trim): $17.8 \mathrm{ft}$. above ground.

(3) Carefully estimate average taper from b.h. to top of first log by use of plumb bob. Best to do this from uphill side of tree so that eye is about level with point midway between $b . h$. and top of log. Average oneside taper estimated to be 3.0 in.

(4) Then, $30.0-2(3.0)=24.0$ in. $0 . b$. top first log. From Fig. 5, bark thickness is 3.0 in.

$$
\frac{24.0-3.0}{30.0} \times 100=70=\text { form class. }
$$

The curve of form class over $\mathrm{a}_{\mathrm{b}} \mathrm{b} . \mathrm{h}$, for all the data used is shown in Fig. 7. The average of all trees was very close to 70 . 


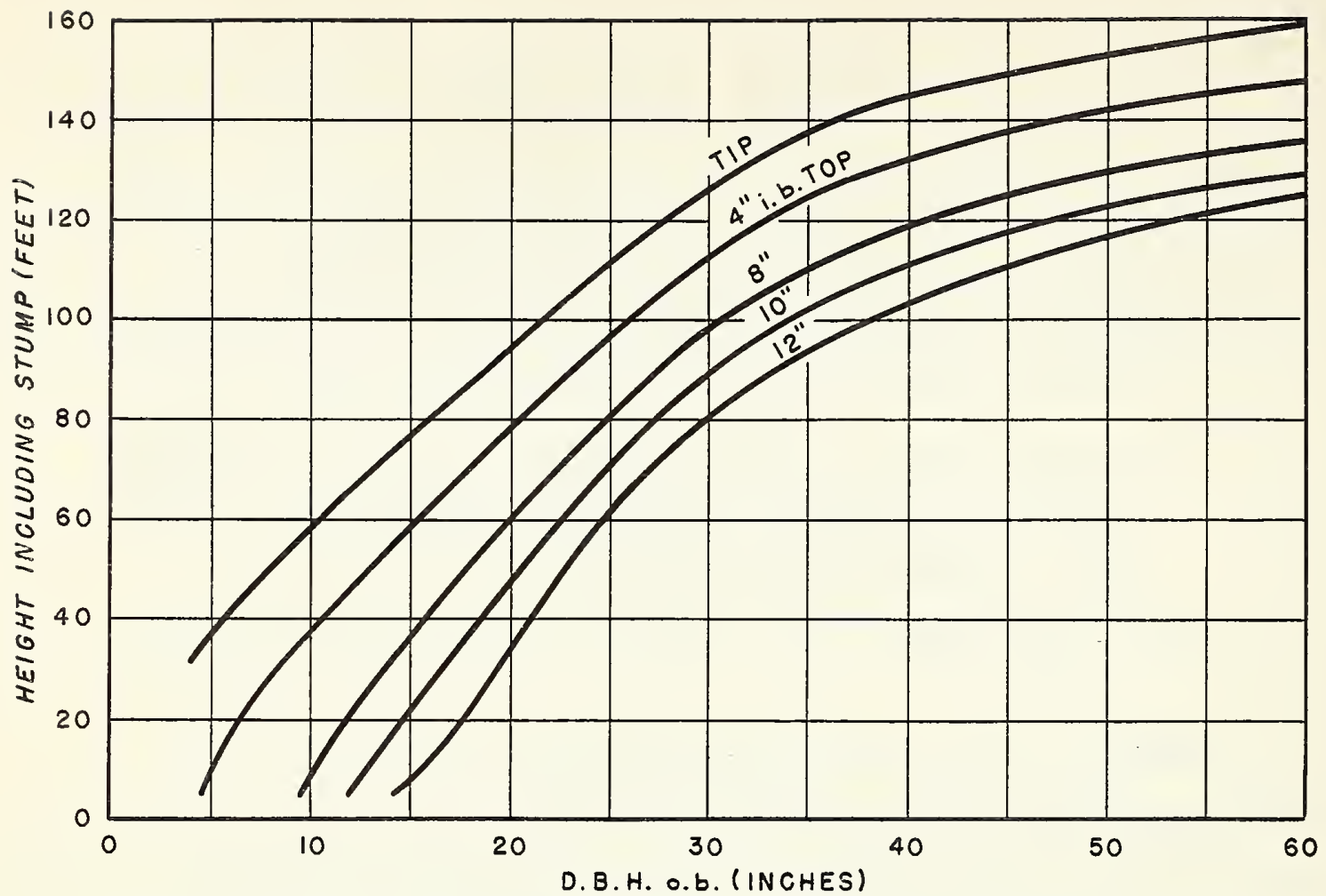

Fig.1 - Height to tip and to fixed top of $4,8,10$, and 12 inches, by d.b.h. Based on 145 trees used in preparing the volume tables.

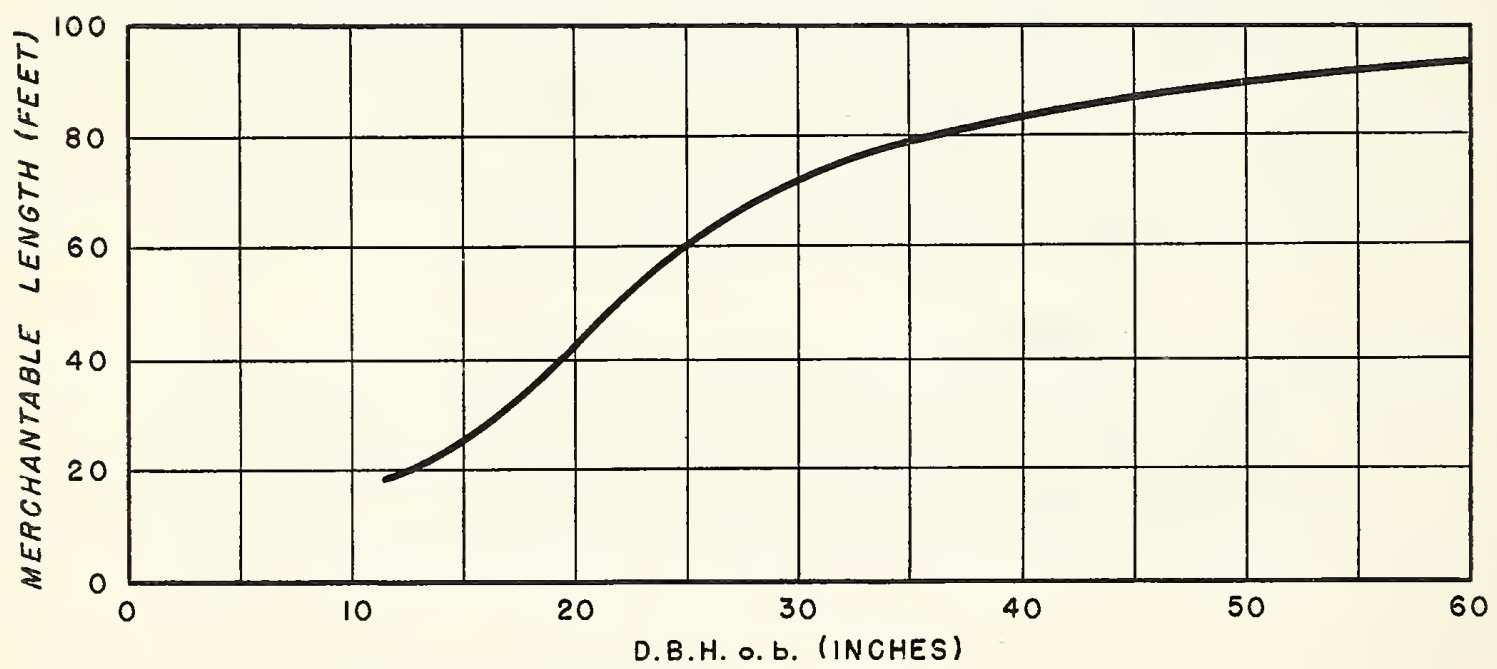

Fig.2 - Merchantable length by d.b.h. Based on 118 trees use in preparing the volume tables. 


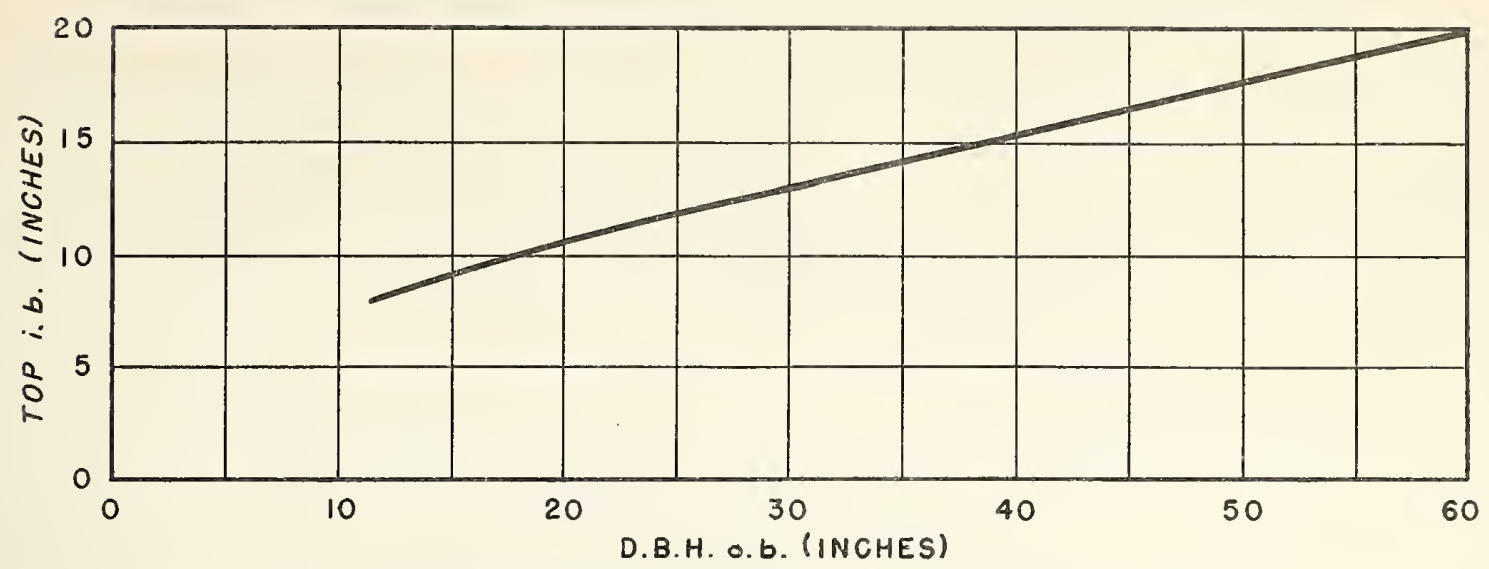

Fig. 3 - Top utilization by d.b.h. classes. (Based on Forest Service data for other species, 1945)

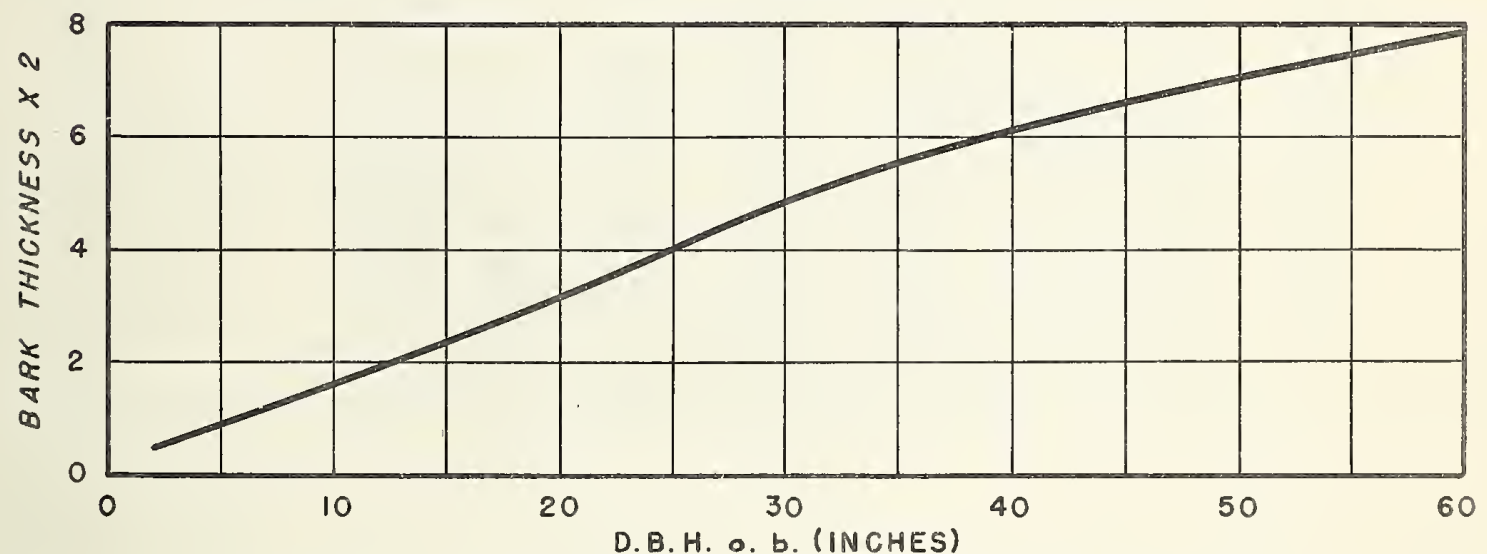

Fig. 4- Double bark thickness in inches at B.H. by d.b.h. outside bark. (Based on 149 trees) 


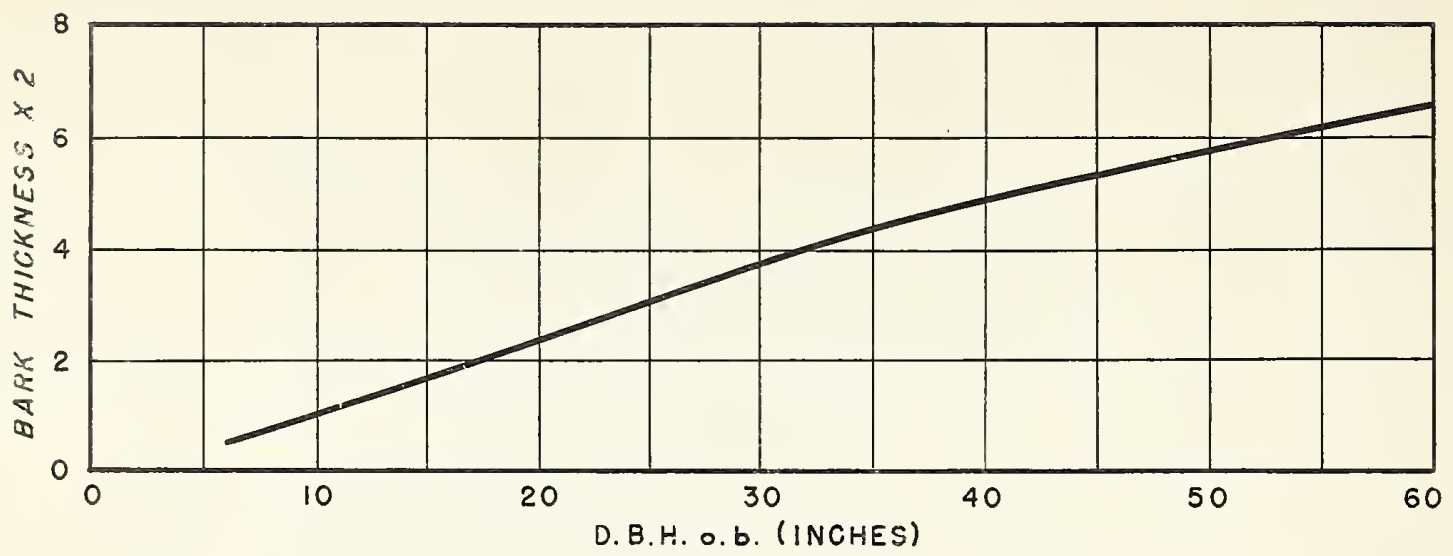

Fig.5 - Double bork thickness in inches at top of first 16 -foot $\log$ by d.b.h. (Based on 149 trees)

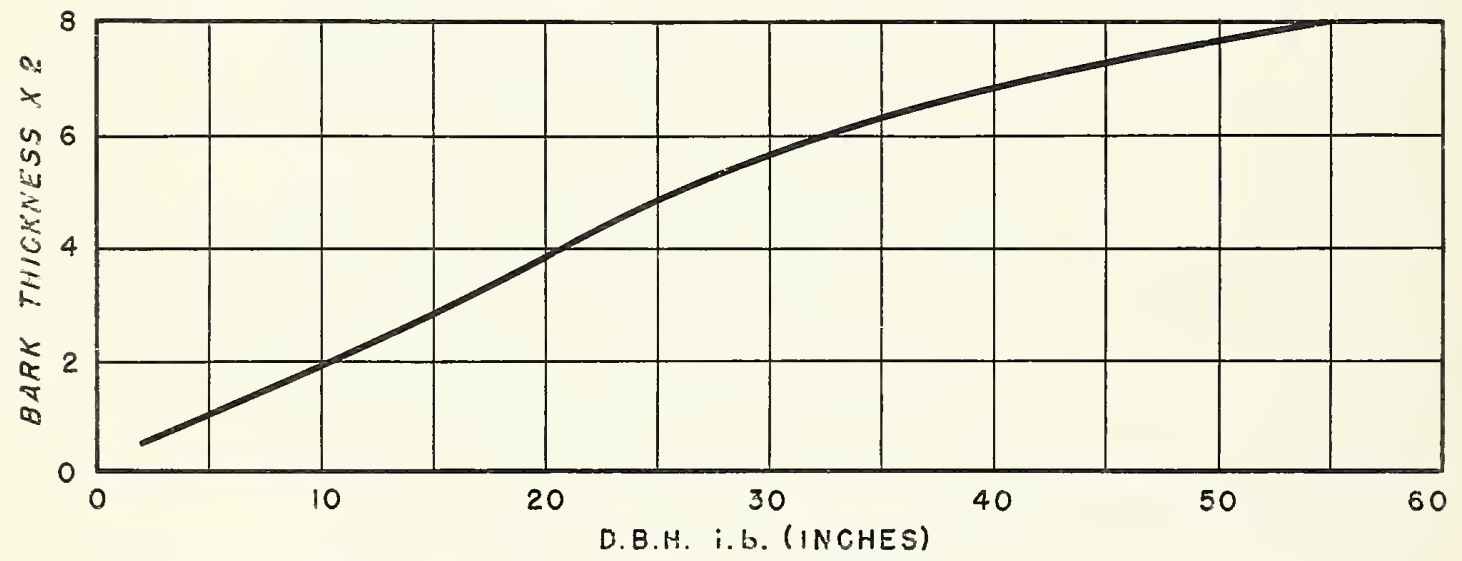

Fig.6 - Double bark thickness in inches af B.H. by d.b.h. inside bork. (Based on 149 trees)

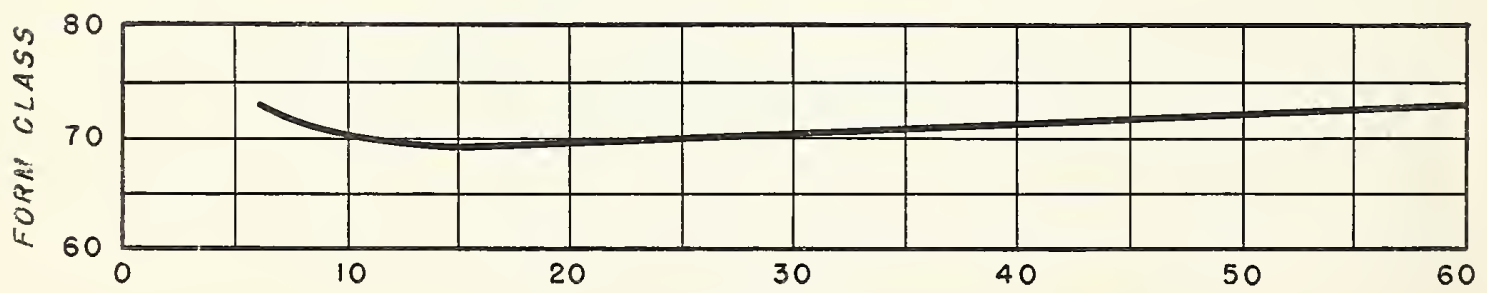

D.B.H. O.b. (INCHES)

Fig.7 - Form class by d.b.h. (Based on 150 trees) 
Table 1.- Form-class Volume Table for Second-growth Redwood

BOARD FEET - SCRIBNER RUI.E

Form Class 70

\begin{tabular}{|c|c|c|c|c|c|c|c|c|c|c|c|c|}
\hline $\begin{array}{c}\text { D.B.H.: } \\
\text { in : }\end{array}$ & & $\mathrm{He}$ & ght is & numb & $r$ of & $3-f$ & ot 108 & S uti & ized & & $\begin{array}{l}\text { Minimum } \\
\text { :top } d . i b\end{array}$ & $\begin{array}{l}\text { :Basís } \\
\text { : No of }\end{array}$ \\
\hline inches: & 1 & 2 & 3 & 4 & 5 & 6 & 7 & 8 & 9 & 10 & :utilized & :trees \\
\hline 12 & 37 & 72 & 105 & & & & & & & & 8 & 9 \\
\hline 14 & 50 & 97 & 142 & & & & & & & & 9 & 13 \\
\hline 16 & 65 & 125 & 183 & 240 & & & & & & & 10 & 17 \\
\hline 18 & 82 & 157 & 230 & 302 & 372 & & & & & & 10 & 9 \\
\hline 20 & 101 & 193 & 282 & 370 & 456 & $54]$. & & & & & 11 & 8 \\
\hline 22 & 121 & 232 & 340 & 445 & 549 & 651 & & & & & 11 & 6 \\
\hline 2.4 & 143 & 2.75 & 402 & 526 & 649 & 770 & 890 & & & & 12 & 4 \\
\hline 26 & & 321 & 469 & 615 & 758 & 900 & .1040 & & & & 12 & 8 \\
\hline 28 & & 370 & 542 & 710 & 875 & 1038 & 1200 & 1360 & & & 13 & 10 \\
\hline 30 & & 423 & 619 & 811 & 1000 & 1187 & 1372 & 1555 & & & 13 & 3 \\
\hline 32 & & 479 & 701 & 919 & 1133 & 1345 & 1554 & 1762 & & & 14 & 6 \\
\hline 34 & & 539 & 789 & 1033 & 1274 & 1512 & 1748 & 1981 & & & 14 & 8 \\
\hline 36 & & 602 & 881 & 1154 & 14.23 & 1689 & 1952 & 2213 & 2472 & 2729 & 15 & 4 \\
\hline 38 & & 668 & 978 & 1282 & 1580 & 1875 & 2168 & 2457 & 2745 & 3030 & 15 & 4 \\
\hline 40 & & 738 & 1080 & 1415 & 1745 & 2071 & 2394 & 2714 & 3031 & 3346 & 15 & 2 \\
\hline 42 & & & 1187 & 1556 & 1918 & 2276 & 2631 & 2983 & 3331 & 3678 & 16 & 1 \\
\hline 44 & & & 1299 & 1702 & 2099 & 2491 & 2879 & 3264 & 3645 & 4025 & 16 & 3 \\
\hline 46 & & & 1416 & 1855 & 2288 & 2715 & 3138 & 3557 & 3973 & 4386 & 17 & 1 \\
\hline 48 & & & & 2014 & 2484 & 2948 & 3407 & 3863 & 431.4 & 4763 & 17 & 1 \\
\hline 50 & & & & 2180 & 2688 & 3190 & 3687 & 4180 & 4669 & 5155 & 18 & - \\
\hline 52 & & & & 2352 & 2900 & 3442 & 3978 & 4510 & 5037 & 5561 & 18 & - \\
\hline 54 & & & & 2530 & 3120 & 3703 & 4280 & 4852 & 5419 & 5983 & 18 & - \\
\hline 56 & & & & 2715 & 3348 & 3973 & 4592 & 5206 & 5815 & 6419 & 19 & - \\
\hline 58 & & & & 2906 & 3583 & 4253 & 4915 & 5572 & 6223 & 6871 & 19 & - \\
\hline 60 & & & & 3103 & 3826 & 4541 & 5248 & 5950 & 6646 & 7337 & 20 & $I$ \\
\hline rees & 22 & 33 & 12 & 25 & 17 & 8 & 1 & - & - & - & & 118 \\
\hline
\end{tabular}

Stump height 18" on uphill side.

Block indicates extent of basic data.

Form Class: Diameter inside bark at top of first 16.3-foot log divided by diameter outside bark at breast height, the result being multiplied by 100. Table above is for the average Form Class of the sample trees. Factors in the tabulation on the reverse side are to be used to get volumes for other Form classes.

The equations from which the tabled volumes were obtained are as follows:

Logarithm b.f. vol. = 1.936,011 (Iogarithm d.b.h. in inches) $+0.939,164$

(logarithm utilized length in feet) $+0.009,961$ (form class) $-2.351,894$. Average deviation of individual tree volumes from values estimated by the equation is 3.9 percent.

Aggregate difference: Estimated values 0.19 percent low. 


\section{MULTIPLIERS FOR OTHER FORM CIASSES}

Form class is 100 times d.i.b. top first log divided by d.b.h. o.b. Factors by which to multiply volumes in Form Class 70 table to obtain volumes for other form classes:

\begin{tabular}{ccccccccccc}
\hline Form Class: & & \multicolumn{1}{c}{ (Units) } \\
(Tens) & 0 & 1 & 2 & 3 & 4 & 5 & 6 & 7 & 8 & 9 \\
\hline 5 & 0.63 & 0.65 & 0.66 & 0.68 & 0.69 & 0.71 & 0.73 & 0.74 & 0.76 & 0.78 \\
6 & 0.80 & 0.81 & 0.83 & 0.85 & 0.87 & 0.89 & 0.91 & 0.93 & 0.96 & 0.98 \\
7 & 1.00 & 1.02 & 1.05 & 1.07 & 1.10 & 1.12 & 1.15 & 1.17 & 1.20 & 1.23 \\
8 & 1.26 & 1.29 & 1.32 & 1.35 & 1.38 & 1.41 & 1.44 & 1.48 & 1.51 & 1.55 \\
\hline
\end{tabular}

Exanple: Volume of $36 ", 5-\log$ tree of form class 78 is $1423 \times 1.20=1708$.

Prepared by:

Division of Forest Economics

California Forest and Range Experiment Station

Forest Service, U. S. Department of Agriculture

A. A. Hasel

February 1950

Aditional copies of this table may be obtained from the California Forest and Range Experiment Station, Box 245, Berkeley 1, California. 
Table 2. - Form-class Volume Table for Second-growth Redwood

BOARD FEET - INTERNATIONAI 1/4-INCH RULE

Form Class 70

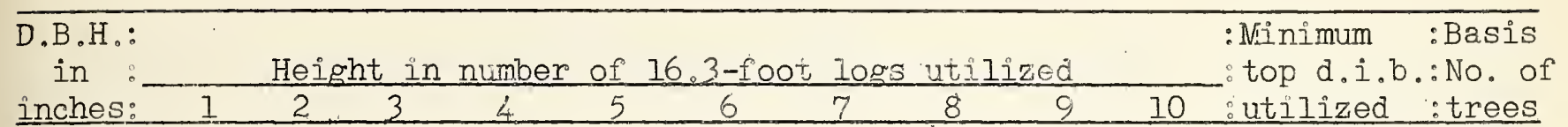

\begin{tabular}{|c|c|c|c|c|c|c|c|c|c|c|c|c|}
\hline 12 & 44 & 83 & 122 & & & & & & & & 8 & 9 \\
\hline 14 & 58 & 111 & 162 & & & & & & & & 9 & 13 \\
\hline 16 & 74 & 142 & 208 & 272 & & & & . & & & 10 & 17 \\
\hline 18 & 92 & 177 & 258 & 339 & 418 & & & & & & 10 & 9 \\
\hline 20 & 112 & 215 & 314 & 411 & 507 & 602 & & & & & 11 & 8 \\
\hline 22 & 133 & 256 & 374 & 491 & 605 & .718 & & & & & 11. & 6 \\
\hline 24 & 157 & 301 & 440 & 576 & 711 & 843 & 975 & & & & 12 & 4 \\
\hline 26 & & 348 & 510 & 668 & 824 & 978 & 1130 & & & & 12 & 8 \\
\hline 28 & & 400 & 585 & 766 & 945 & 1121 & 1296 & 1469 & & & 13 & 10 \\
\hline 30 & & 454 & 664 & 870 & 1073 & 1274 & 1472 & 1669 & & & 13 & 3 \\
\hline 32 & & 512 & 749 & 9,1 & 1209 & 1435 & 1659 & 1880 & & & 14 & 6 \\
\hline 34 & & 572 & 837 & 1097 & 1353 & 1605 & 1855 & 2103 & & & 14 & 8 \\
\hline 36 & & 636 & 931 & 1219 & 1504 & 1784 & 2062 & 2338 & 2611 & 2883 & 15 & 4 \\
\hline 38 & & 703 & 1028 & 1347 & 1662 & 1972 & 2279 & 2583 & 2885 & 3186 & 15 & 4 \\
\hline 40 & & 773 & 1131 & 1481 & 1827 & 2168 & 2506 & 2840 & 3172 & 3502 & 15 & 2 \\
\hline 42 & & & 1237 & 1621 & 1999 & 2372 & 2742 & 3108 & 3472 & 3833 & 16 & 1 \\
\hline 44 & & & 1349 & 1767 & 2179 & 2586 & 2988 & 3387 & 3784 & 4177 & 16 & 3 \\
\hline 46 & & & 1464 & 1918 & 2365 & 2807 & 3244 & 3677 & 4108 & 4535 & 17 & 1 \\
\hline 48 & & & & 2075 & 2559 & 3037 & 3510 & 3978 & 4444 & 4906 & 17 & 1 \\
\hline 50 & & & & 2238 & 2759 & 3275 & 3785 & 4290 & 4792 & 5290 & 18 & - \\
\hline 52 & & & & 2406 & 2967 & 3521 & 4069 & 4613 & 5152 & 5688 & 18 & - \\
\hline 54 & & & & 2580 & 3181 & 3775 & 4363 & 4946 & 5525 & 6099 & 18 & - \\
\hline 56 & & & & 2759 & 3402 & 4038 & 4667 & 5290 & 5909 & 6523 & 19 & - \\
\hline 58 & & & & 29 lobe & 3630 & 4303 & 4979 & 5645 & 6305 & 6960 & 19 & - \\
\hline 60 & & & & 3135 & 3865 & 4587 & 5301 & 6010 & 6712 & 7413 & 20 & 1 \\
\hline & 22 & 33 & 12 & 25 & 17 & 8 & 1 & - & - & - & & 118 \\
\hline
\end{tabular}

Stump height 18" on uphil1 side.

Block indicates extent of vasic data.

Form Class: Diameter inside bark at top of first 1.6.3-foot log divided by diameter outside bark at breast height, the result being multiplied by 100. Table above is for the average Form Class of the sample trees. Factors in the tabulation on the reverse side are to be used to get volumes for other Form. Classes.

The equations from which the tabled volumes were obtained are as follows: Logarithm b.f. vol. $=1.848,446$ (logaritinm a.b.i. in inches) $+0.939,005$

(logarithm utilized length in feet) + 0.009,548 (form class) - 2.162,583. Average deviation of individual tree volumes from values estimated by the equation is 3.0 percent

Aggregate difference: Estimated values 0.19 percert low 


\section{MULTIPLIERS FOR OTHER FORM CLASSES}

Form class is 100 times $d . i . b$. top first log divided by $d . b . h .0 . b$. Factors by which to multiply volumes in Form Class 70 table to obtain volumes for other form classes:

\begin{tabular}{|c|c|c|c|c|c|c|c|c|c|c|}
\hline \multirow{2}{*}{$\begin{array}{c}\text { Form Class } \\
\text { (T'ens) }\end{array}$} & \multicolumn{10}{|c|}{ (Units) } \\
\hline & 0 & 1 & 2 & 3 & 4 & 5 & 6 & 7 & 8 & 9 \\
\hline 5 & 0.64 & 0.66 & 0.67 & 0.69 & 0.70 & 0.72 & 0.74 & 0.75 & 0.77 & 0.79 \\
\hline 6 & 0.80 & 0.82 & 0.84 & 0.86 & 0.88 & 0.90 & 0.92 & 0.94 & 0.96 & 0.98 \\
\hline 7 & 1.00 & 1.02 & 1.04 & 1.07 & 1.09 & 1.12 & 1.14 & 1.17 & 1.19 & 1.22 \\
\hline 8 & 1.25 & 1.27 & 1.30 & 1.33 & 1.36 & 1.39 & 1.42 & 1.45 & 1.49 & 1.52 \\
\hline
\end{tabular}

Example: Volume of 36", 5-log tree of form class 78 is $1504 \times 1.19=1790$.

Prepared by:

Division of Forest Economics

California Forest and Range Experiment Station

Forest Service, U. S. Department of Agriculture

A. A. Hasel

February 1950

Additional copies of this table may be obtained from the California Forest and Range Experiment Station, Box 245, Berkeley 1, California. 
Table 3. - Form-class Volume Table for Second-growth Redwood

CUBIC FEET IN SAHLOG POPTION

Form Class 70

\begin{tabular}{|c|c|c|c|c|c|c|c|c|c|c|c|c|}
\hline $\begin{array}{l}\text { D.B.H.: } \\
\text { in : }\end{array}$ & & & ight i & numbe & of 16 & $3-100 t$ & logs & tiliz & & & $\begin{array}{l}\text { :Minimum } \\
: \text { top d.i.b. }\end{array}$ & $\begin{array}{l}\therefore \text { Basis } \\
\therefore \text { No of }\end{array}$ \\
\hline inches: & 1 & 2 & 3 & 4 & 5 & 6 & 7 & 8 & 9 & 10 & :utilized & 1:trees \\
\hline 12 & 8.1 & 13.4 & 18.7 & & & & & & & & 8 & 9 \\
\hline 14 & 10.8 & 17.9 & 25.0 & & & & & & & & 9 & 13 \\
\hline 16 & 13.9 & 23.1 & 32.2 & 40.1 & & & & & & & 10 & 17 \\
\hline 18 & 17.3 & 28.8 & 40.2 & 50.1 & 59.4 & & & & & & 10 & 9 \\
\hline 20 & 21.1 & 35.1 & 49.0 & 61.1 & 72.4 & 83.3 & & & & & 11 & 8 \\
\hline 22 & 25.2 & 42.0 & 58.6 & 73.0 & 86.7 & 99.7 & & & & & 11 & 6 \\
\hline 24 & 29.7 & 49.4 & 69.0 & 86.0 & 102. & 117. & 132. & & & & 12 & 4 \\
\hline 26 & & 57.4 & 80.2 & $1.00 \%$ & 119. & 136. & 154. & & & & 12 & 8 \\
\hline 28 & & 66.0 & 92.2 & 115. & 136. & 157. & 177 . & 196 。 & & & 13 & 10 \\
\hline 30 & & 75.2 & 105. & 131. & 155. & 179. & 201 . & 223 & & & 13 & 3 \\
\hline 32 & & 84.9 & 119. & $1<8$ 。 & 175. & 202. & 227. & 251. & & & 14 & 6 \\
\hline 34 & & 95.1 & 133. & 166. & 196. & 226. & 254 。 & 282. & & & 14 & 8 \\
\hline 36 & & 106. & 148. & 184. & 219. & 252. & 283. & 314. & 343. & $372 。$ & 15 & 4 \\
\hline 38 & & 117. & 164. & 204 . & 242. & 279. & 323. & 347. & 380 。 & 412. & 15 & 4 \\
\hline 40 & & 129. & 180. & 225. & 267. & 307. & 345. & 382. & 419. & 454. & 15 & 2 \\
\hline 42 & & & 198. & 246. & 292. & 336. & 378. & 419. & 459. & 497. & 16 & 1 \\
\hline 44 & & & 216. & 269. & 319. & 367. & 413. & 458. & 501. & 543. & 16 & 3 \\
\hline 46 & & & 234. & $292 。$ & 347. & 399. & 449. & 497. & 544. & 590. & 17 & 1 \\
\hline 48 & & & & 317. & 376. & $\leq 32$. & 486. & 539. & 590 。 & 639. & 17 & 1 \\
\hline 50 & & & & 342. & 206 . & 467. & 525. & 582. & 637. & 690 。 & 18 & - \\
\hline 52 & & & & 368. & 437. & 502. & 565. & 625. & 686. & 743. & 18 & - \\
\hline 54 & & & & 395. & 469. & 539. & 607. & 672. & 736. & 798. & 18 & - \\
\hline 56 & & & & 423. & 502. & 578. & 650 。 & 720. & 788. & 854. & 19 & - \\
\hline 58 & & & & 452. & 536. & 617. & 694 . & 769. & 842. & 913. & 19 & - \\
\hline 60 & & & & 482. & 572. & 658. & 740 . & 820. & 897. & 973. & 20 & 1 \\
\hline Trees & 22 & 33 & $\mathrm{i} 2$ & 25 & 17 & 8 & 1 & - & - & - & & 118 \\
\hline
\end{tabular}

Volume excluding bark.

Stump height 18" on uphill side.

Block indicates extent of basic data.

Form Class: Diameter inside bark at top of first 16.3-foot log divided by diameter outside bark at breast height, the result being multiplied by 100. Table above is for the average Form Class of the sample trees. Factors in the tabulation on the reverse sice are to be used to get voiumes for other Foril classes.

The equations from which the tabled volumes were obtained are as follows:

Logarithm cubic volume sawlog portion $=1.880,403$ (logarithm d.b.h. in inches) $+0.766,659$ (logarithm utilized length in feet) $+0.008,545$ (form class) $-2.649,799$.

Average deviation of individual tree volumes from values estimated by the equation is 3.5 percent.

Aggregate difference: Estimated values 0.10 percent low. 


\section{MULTIPLIERS FOR OTHER FORM CLASSES}

Form class is 100 times $\mathrm{d} \cdot \mathrm{i} \cdot \mathrm{b}$. top first log divided by $\mathrm{d} \cdot \mathrm{b} \cdot \mathrm{h}$. o.b. Factors by which to multiply volumes in Form Class 70 table to obtain volumes for other form classes:

\begin{tabular}{|c|c|c|c|c|c|c|c|c|c|c|}
\hline \multirow{2}{*}{$\begin{array}{c}\text { Form Class } \\
\text { (Tens) }\end{array}$} & \multicolumn{10}{|c|}{ (Units) } \\
\hline & 0 & 1 & 2 & 3 & 4 & 5 & 6 & 7 & 8 & 9 \\
\hline 5 & 0.67 & 0.69 & 0.70 & 0.72 & 0.73 & 0.74 & 0.76 & 0.77 & 0.79 & 0.81 \\
\hline 6 & 0.82 & 0.84 & 0.85 & 0.87 & 0.89 & 0.91 & 0.92 & 0.94 & 0.96 & 0.98 \\
\hline 7 & 1.00 & 1.02 & 1.04 & 1.06 & 1.08 & 1.10 & 1.13 & 1.15 & 1.17 & 1.19 \\
\hline 8 & 1.22 & 1.24 & 1.27 & 1.29 & 1.32 & 1.34 & 1.37 & 1.40 & 1.42 & 1.45 \\
\hline
\end{tabular}

Example: Volume of $36 ", 5-\log$ tree of form class 78 is $219 \times 1.17=256$.

Prepared by:

Division of Forest Economics

California Forest and Range Experiment Station

Forest Service, U. S. Department of Agriculture

A. A. Hasel

February 1950

Additional copies of this table may be obtained from the California Forest and Range Experiment Station, Box 245, Berkeley 1, California. 


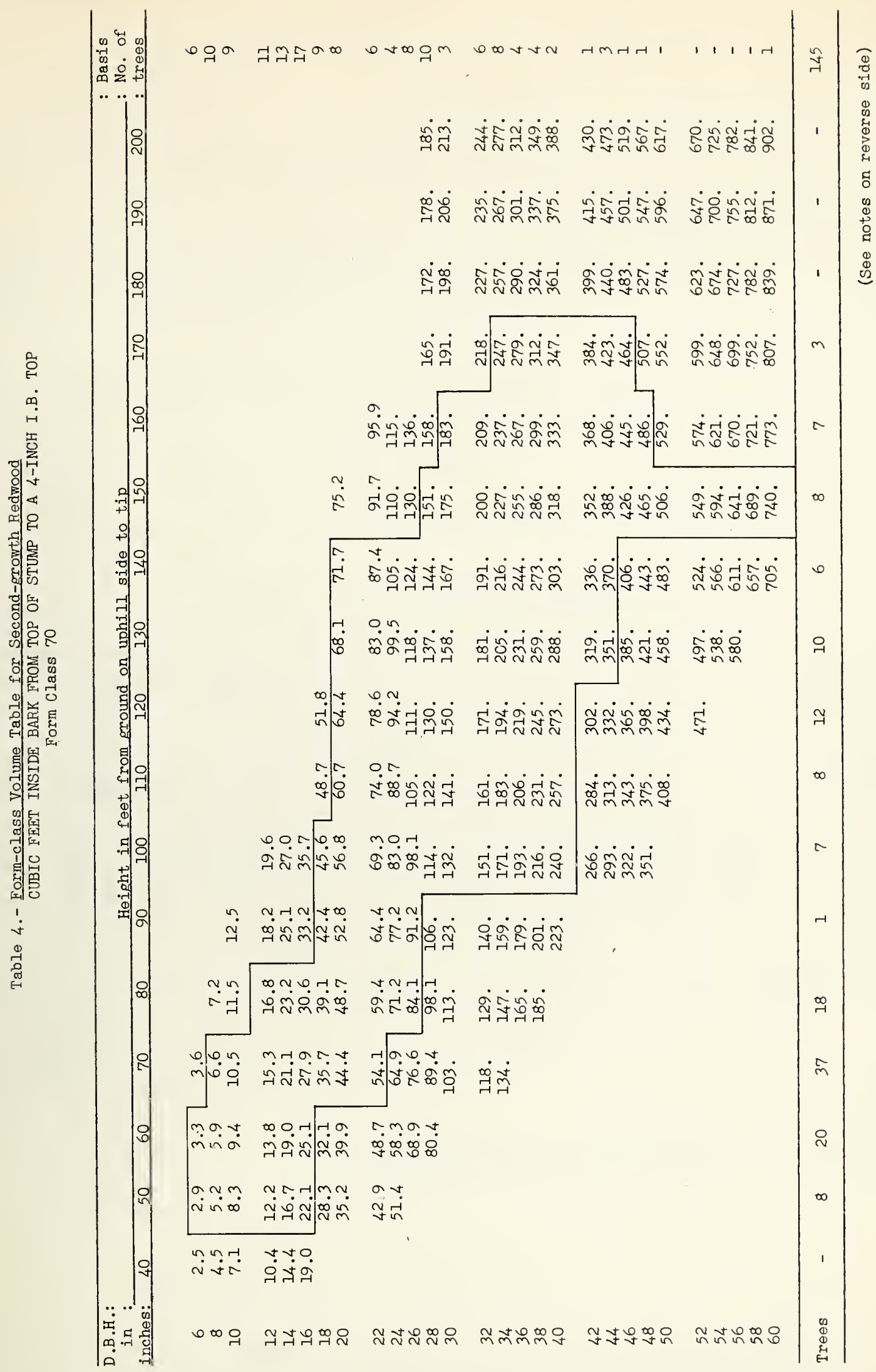


Volume excludes bark and stump.

Stump heights measured from uphill side: 13" for 6" d.b.h., 15" for 8" d.b.h., 17" for $10 " \mathrm{~d}, \mathrm{~b} . \mathrm{h} .$, and 18 " for $12 " \mathrm{~d} . \mathrm{b} . \mathrm{h}$. and larger.

Blcck indicates extent of basic data.

Form Class: Diameter inside bark at top of first 15.3-foot log divided by diameter outside bark at breast height, the result being multiplied by 100 . Table on reverse side is for the average Form Class of the sample trees. Factors in the tabulation below are to be used to get volumes for other Form Classes.

The equations from which the tabled volumes were obtained are as follows:

Iogarithm cubic volume to 4 inches $i . b .=2.081,127$ (logarithm $d . b . h$. in inches) $+0.691,137$ (logarithm total height in feet) $+0.010,285$ (form class) - 3.055, 417.

Average deviation of individual tree volumes from values estimated by the equation is 6.6 percent.

Aggregate difference: Estimated values 0.01 percent high.

\section{MULTIPLIERS FOR OTHER FORM CLASSES}

Form class is 100 times d.i.b. top first log divided by d.b.h. o.b. Factors by which to multiply volumes in Form Class 70 table to obtain volumes for other form classes:

\begin{tabular}{ccccccccccc}
\hline $\begin{array}{c}\text { Form Class : } \\
\text { (Tens) }\end{array}$ & 0 & 1 & 2 & 3 & 4 & 5 & 6 & 7 & 8 & 9 \\
\hline 5 & 0.62 & 0.64 & 0.65 & 0.67 & 0.68 & 0.70 & 0.72 & 0.74 & 0.75 & 0.77 \\
6 & 0.79 & 0.81 & 0.83 & 0.85 & 0.87 & 0.89 & 0.91 & 0.93 & 0.95 & 0.98 \\
7 & 1.00 & 1.02 & 1.05 & 1.07 & 1.10 & 1.13 & 1.15 & 1.18 & 1.21 & 1.24 \\
8 & 1.27 & 1.30 & 1.33 & 1.36 & 1.39 & 1.43 & 1.46 & 1.50 & 1.53 & 1.57
\end{tabular}

Example: Volume of 36", 150-foot tree of form class 78 is $255 \times 1.21=308$.

Prepared by:

Division of Forest Economics

California Forest and Range Experiment Station

Forest Service, U. S. Department of Agriculture

Additional copies of this table may be obtained from the California Forest and Range Experiment Station, Box 245, Berkeley 1, California. 